\title{
Anterior meatuscopy is more reliable than a sniff test for predicting nasal patency before transnasal endoscopy
}

Author

Institutions
Chi-Tan $\mathrm{Hu}^{1,2}$

${ }^{1}$ Division of Gastroenterology, Department of Internal Medicine, Buddhist Tzu Chi General Hospital and Tzu Chi University, Hualien, Taiwan

${ }^{2}$ Graduate Institute of Clinical Medicine, School of Medicine, Tzu Chi University, Hualien, Taiwan

\section{Bibliography}

DOI http://dx.doi.org/

10.1055/s-0034-1392772

Published online: 6.10.2015

Endoscopy International Open 2015; 03: E538-E546

(c) Georg Thieme Verlag KG

Stuttgart · New York

E-ISSN 2196-9736

\section{Corresponding author} Chi-Tan Hu, MD, PhD

Division of Gastroenterology Department of Internal

\section{Medicine}

Buddhist Tzu Chi Hospital and

Tzu Chi University

No 707 Section 3

Chung Yang Road

Hualien (970)

Taiwan

Fax: +886-3-8567043

chitan.hu@msa.hinet.net
Background and study aims: Unsedated transnasal esophago-gastro-duodenoscopy (UT-EGD) has been performed for at least two decades but many techniques related to the procedure have yet to be standardized. We aimed to develope a simple endoscopic method, anterior meatuscopy, to replace the sniff test for selecting the most patent nasal meatus before UT-EGD. We hypothesized that access to the common nasal meatus (CNM), if confirmed by anterior meatuscopy, was a safer route than the inferior (INM) and middle nasal meatuses (MNM) because it would result in reduced epistaxis, nasal pain, and nasal discharge. Patients and methods: We used anterior meatuscopy and an endoscopic meatus scoring scale (EMSS) to identify the optimal meatus for insertion. We evaluated the EMSS frequency distributions for the INM, CNM, and MNM and the reliability of a sniff test in 1,000 consecutive patients. The adverse effects of passing through the CNM versus the INM and MNM, respectively, were compared.

\section{Introduction \\ $\nabla$}

In recent years, unsedated transnasal esophagogastro-duodenoscopy (UT-EGD) has been used for diagnostic [1,2] and therapeutic purposes such as percutaneous endoscopic gastrostomy [3], enteral tube placement [4], nasobiliary tube drainage [5, 6] and polypectomy [7]. The satisfactory safety and tolerance profiles make UT-EGD an alternative to peroral conventional EGD with or without sedation $[1,8]$. There is a trend toward performing transnasal laryngoscopy [9], transnasal esophagoscopy [10] (especially for screening Barrett's esophagus $[2,10,11])$, and UT-EGD in the office $[11,12]$. However, many techniques related to UT-EGD are not standardized [13]. One important step, the selection of the optimal meatus insertion route for UT-EGD, has not been explored.
Results: In the INM and MNM, the EMSS frequencies were grade I $>$ II $>$ III $>0$ whereas those in the CNM were grade II $>$ I $>0>$ III. A sniff test was not reliable, given its low sensitivity (63.3\%), specificity $(67.3 \%)$, positive predictive value $(69.1 \%)$, negative predictive value (61.4\%), and accuracy (65.2\%). Although only $9.2 \%$ of our patients had grade III anterior-to-posterior CNMs, passage through this spacious CNM rather than through the MNM or the INM could significantly reduce epistaxis, nasal pain, and nasal discharge.

Conclusions: Anterior meatuscopy is more reliable than a sniff test before UT-EGD. Transnasal endoscopic passage through the endoscopically patent CNM, if detected by anterior meatuscopy, may prevent epistaxis and nasal pain and minimize nasal discharge.

\section{License terms}

()(1) $\odot \circledast$
The sniff test is a subjective way to select a nostril insertion site for nasal anesthesia [11 - 16]. A nostril insertion site is not the final meatus insertion route, which is at the endoscopist's discretion. A wrongly selected meatus insertion route may cause insertion failure, nasal pain, nasomucosal injury, or epistaxis [14-17]. Yet there is no study to evaluate the reliability of a sniff test before these transnasal endoscopic procedures. The idea that "looking to select" is better than "sniffing to select" emerged from our study 7 years ago [14]. We have previously described endoscopic-guided aerosolized spray for nasal anesthesia before UTEGD; with an ultrathin transnasal endoscope, the endoscopist searched for the most patent nasal meatus from both sides before applying aerosolized sprays of lidocaine $[14,15]$. To establish a simple endoscopic meatus scoring scale (EMSS), we have further devised a new concept, anterior 

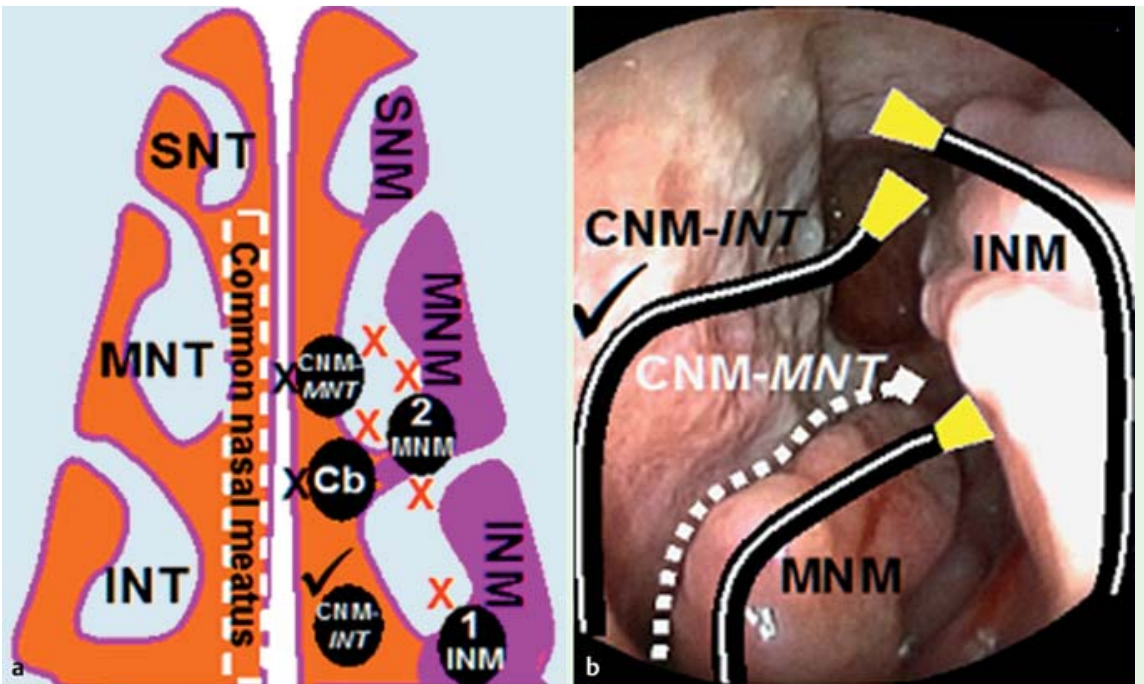

Fig. 1 The common nasal meatus is the route of choice. (a) The commonly recognized meatus for nasal insertion is the INM (1) or the MNM (2). Usually the CNM at the INT level (CNM-INT, $\checkmark)$ is wider than that at the MNT level (CNM-MNT). When passing through the INM, MNM, CNM-MNT level or between the CNM-INT and CNM-MNT (Cb), the endoscope (black circles) may induce mucosal injury or epistaxis (X) through contact with the MNT and/ or INT (red $\mathbf{X}$ ) or the nasal septum (black $\mathbf{X}$ ). (b) Endoscopic view showing that insertion through the CNM-INT level (right nasal cavity) is the safest route $(\checkmark)$. (c) In the same patient, the nasal septum deviation (arrow) nearly completely obstructs the proximal MNM and only the CNM-INT (left nasal cavity) is patent enough for insertion $(\checkmark)$. (d) In another patient, a nasal septal spur (arrow) completely obstructs the distal MNM and only the CNM-INT (right nasal cavity) is patent enough for
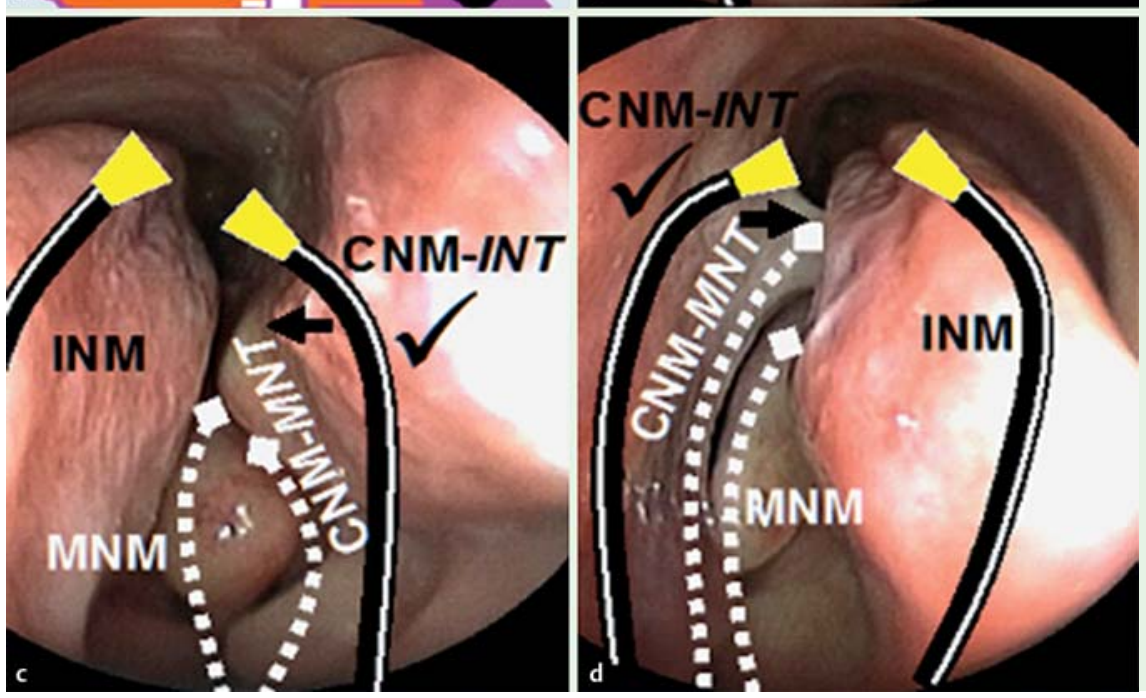
insertion $(\checkmark)$. The dotted lines in (b), (c) and (d) represent routes that are too narrow for transnasal insertion.

meatuscopy, to compare nasal meatus patency scores for each side of the nasal cavity.

Mori et al reported that in their transnasal endoscopic examinations of 1,569 patients, $78 \%$ of nasal bleeding sites were in the middle nasal turbinate (MNT), $15 \%$ in the inferior nasal turbinate (INT), and 6\% in the nasal septum [18]. Their data suggest that the middle nasal meatus (MNM) is the most frequently used tract but the inferior nasal meatus (INM) and common nasal meatus (CNM) are uncommon routes for nasal insertion in Japan. In fact the CNM, which is between the nasal septum and medial walls of the MNT and INT, is neglected by endoscopists. Ignorance of the $\mathrm{CNM}$ is particularly common in endoscopists who are unfamiliar with UT-EGD.

We hypothesized that the CNM, if detected by anterior meatuscopy, was a safer route than the MNM or the INM for avoiding the most common adverse effects of UT-EGD, including epistaxis, nasal pain and nasal discharge [14-16]. Thus, using the transnasal endoscope at hand as a meatuscope, we evaluated patency scores of the nasal meatuses before transnasal endoscopy. We compared the results with the reliability of the sniff test, which has been used for decades. More importantly, we compared the adverse effects of passing through the CNM versus an equally patent MNM and INM.

\section{Patients and methods \\ $\nabla$}

All 1,000 consecutive outpatients with dyspeptic symptoms underwent both a sniff test and an anterior meatuscopy before UTEGD to obtain their respective EMSS. The study was conducted at Buddhist Tzu Chi Hospital between 2011 and 2014, employing an EG530N5 endoscope (Fujinon, Tokyo, Japan). Patients who had prior nasal trauma or surgery, recent or present upper gastrointestinal bleeding, and who were unable to answer questions were excluded from this study. Patients who were allergic to any anesthetic agents were not recruited.

\section{Sniff test}

Instead of the conventional left lateral decubitus position, all patients sat upright in an examination bed. The more patent nostril was selected by the patient inhaling through only one nostril with the other sealed by the examiner's index finger [14].

\section{Anterior meatuscopy}

We have developed an endoscopic method that allow us to look for the optimal meatus insertion route in just seconds. When placed in the anterior nasal cavity, a transnasal endoscope itself can act as a nasoscope (or more precisely a meatuscope) to compare insertability and patencies of the INM, CNM and MNM. Anterior rhinoscopy examines the mucosa and anatomical pa- 

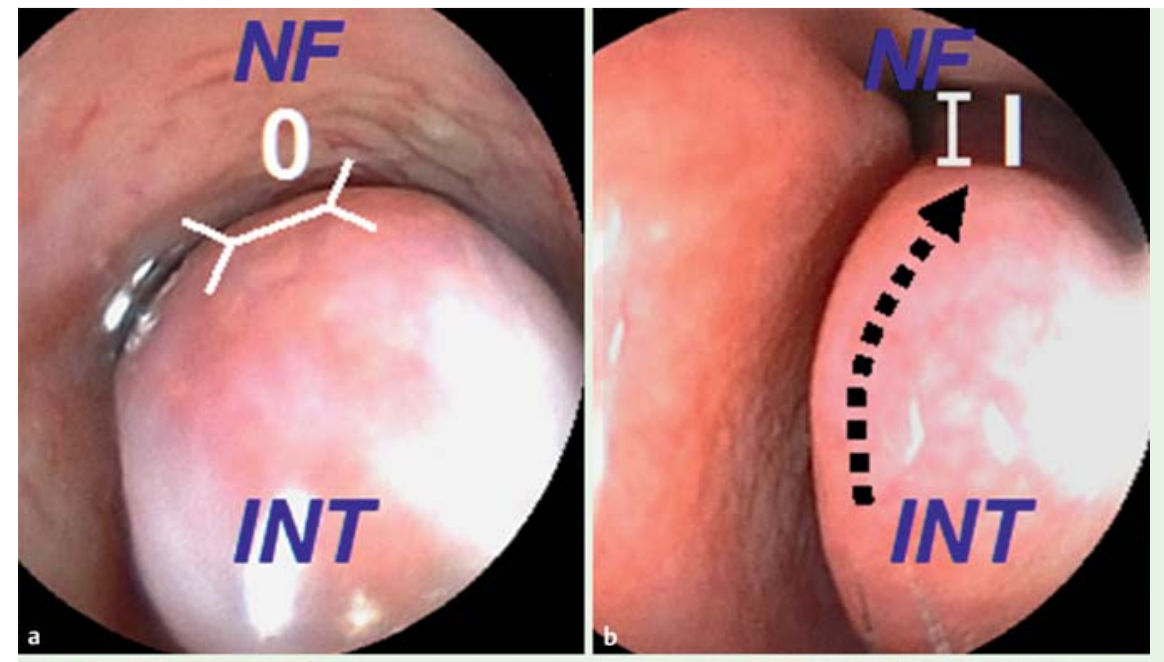

Fig. 2 EMSS of the INM (right). The INM is the space between the inferior aspect of the INT and the nasal floor (NF). (a) grade 0: no space; (b) grade l: only up-sloping INT border (black arrow) and slitlike distant INM are seen; (c) grade II: (i) the upsloping/down-sloping INT border (the curved endoscope) and transition angle (*) plus (ii) the incomplete choana (dotted line) are seen; a nasal septum (NS) spur (arrow) abutting against the posterior MNM; (d) grade III: (i) the posterior insertion of the INT (PI-INT, arrow), (ii) the PI-MNT (arrowhead) and (iii) the complete choana (dotted circle)
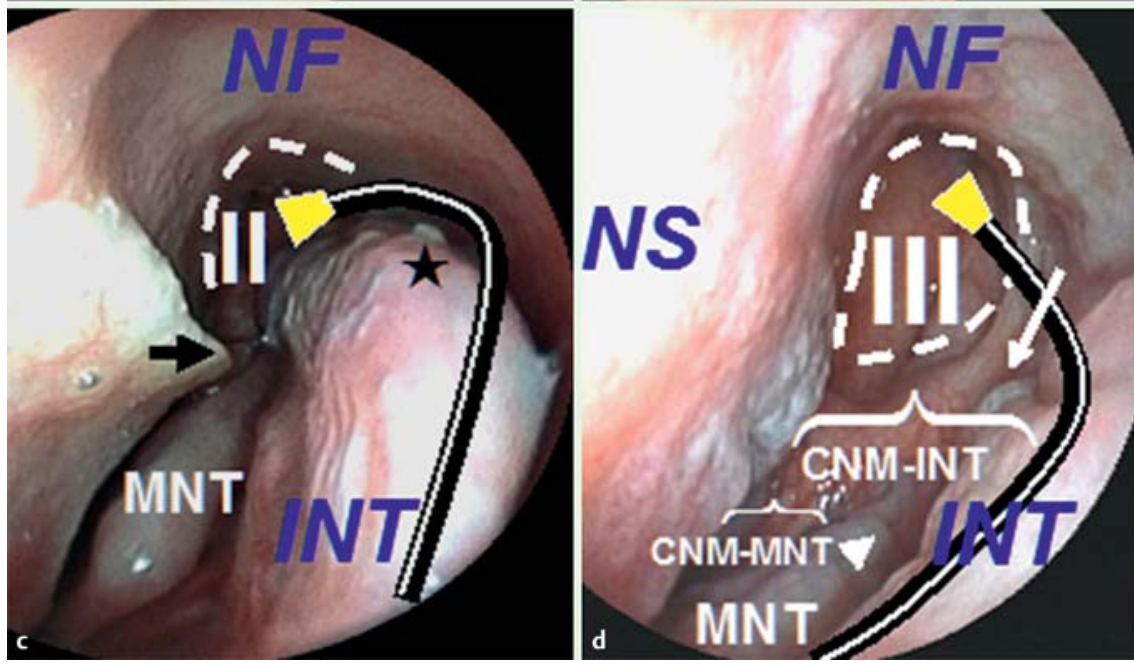
are seen. In contrast to the scope in (c) through the INM, the scope in (d) is along the CNM at the INT level.

thology inside the anterior nasal cavity. In contrast, anterior meatuscopy is simple to perform, accurate for selection of the most patent nasal meatus, and appropriate for all kinds of patients. With anterior meatuscopy, the characteristics of the CNM, INM, and MNM can be more clearly elucidated.

The CNM versus the MNM/INM

The CNM is the space between the nasal septum and "medial" sides of the MNT and INT. In contrast, the routes of the INM or the MNM are along the "inferior" aspects of their corresponding turbinates ( $\bullet$ ig. 1a). The CNM at the MNT height (CNM-MNT level) is usually too narrow to permit passage of a transnasal endoscope ( Fig.1). Compared with the CNM-MNT level, the CNM at the INT height level (CNM-INT level) is wide enough for transnasal endoscope insertion and exertion ( $\bullet$ Fig. 1 and $\bullet$ Fig. 2 d). Thus, in clinical practice, both the anterior and posterior CNMs actually refer to the CNM-INT level.

\section{The MNM versus the INM}

In the nasal cavity, the anterior head of the INT is more anterior than the anterior head of the MNT ( $\bullet$ Fig. $3 c$ ). Sometimes, the anterior head of the INT is hypertrophic to some extent, preventing approach to the inner MNM. Therefore, the anterior CNM-INT level (herein below simply denoted as anterior CNM) is the only gateway for entry to the atrium of the MNM ( Fig.3c and - Fig. $3 \mathrm{~d}$ ), which is the anterior expanded portion of the MNM just above the nasal vestibule. More specifically, the actual pass- by sites of a transnasal endoscope to the MNM comprise the "CNM-atrium-MNM" structures, constituting a straightforward route ( $\bullet$ Fig.3c and $\odot$ Fig.4c). However, endoscopic insertion through the MNM route is most commonly hindered when the INT and the nasal septum abut each other, forming an "endoscope clamp" ( Fig.4c, arrows). Conversely, the endoscopic pathway of the INM is along the curved inferior surface of the INT $(-$ Fig. 2 c) without the need to go through the anterior CNM $(\nabla$ Fig. $3 \mathrm{c}$ ). With either the INM or the MNM route, the up-down angulation movement of the scope tends to cause epistaxis in the inferior surface of the INT or the MNT.

\section{The safest tunnel}

A safe CNM should demonstrate patency anteriorly and posteriorly on endoscopy. Given such a spacious CNM, the up-down angulation movements of an endoscope tip would be less likely to cause injury to the INT or the MNT. Therefore, the anterior-toposterior CNM, if endoscopically patent enough, was hypothesized to be the "safest tunnel" for trans-meatal passage (the scope route shown in $\mathbf{F i g . 2}$ d). In this study, we defined patency scores for the anterior meatuses but not for the posterior ones because anterior meatuscopy principally observes the anterior nasal cavity. We defined an endoscopically patent anterior CNM (a grade III anterior CNM), which allows the scope to be inserted to the atrium of the MNM. The posterior CNM was not defined or evaluated with anterior meatuscopy. Instead, an endoscopically patent posterior CNM was always available in a grade III INM, 

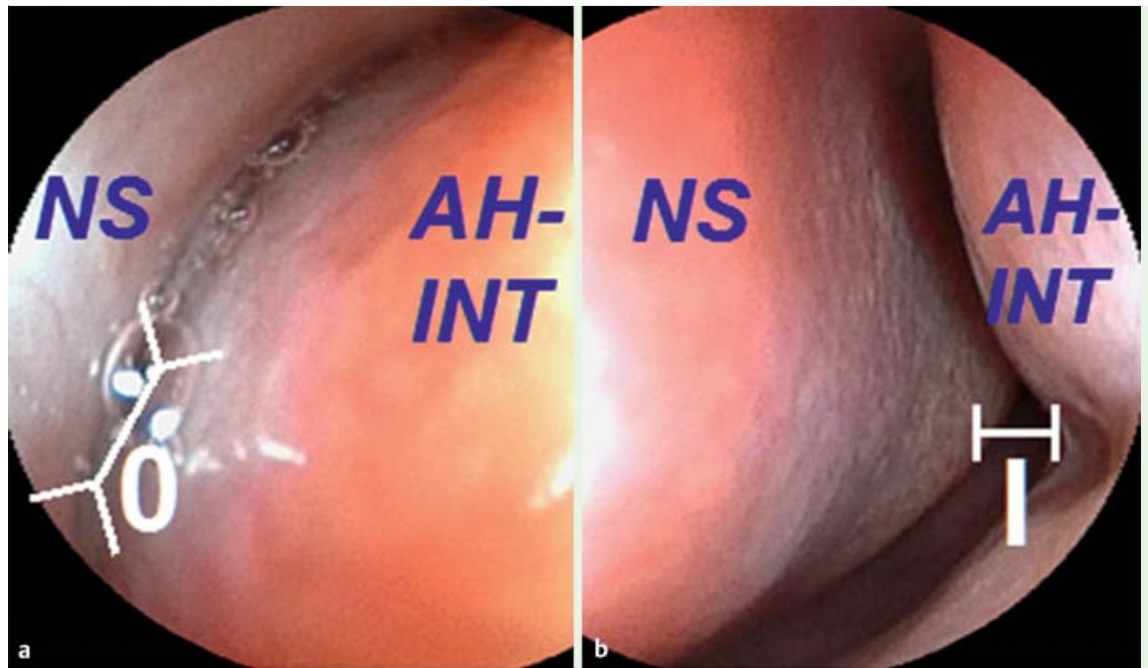

Fig. 3 EMSS of the anterior CNM (right). The anterior CNM is the space between the anterior head of the INT (AH-INT) and the NS. (a) grade 0: no space; (b) grade I: slit-like; (c) grade II: although the anterior head of the MNT (AH-MNT) is seen, the scope in the anterior CNM cannot reach the atrium of the MNM (dotted line region) because of scope resistance and/or nasal discomfort. Thus, with a grade II anterior CNM, EMSS of the inner MNM is regarded as not evaluable; (d) grade III: the scope can reach the atrium of the MNM without scope resistance and/or nasal discomfort. Usually the whole AH-MNT is seen in a grade III anterior CNM. In contrast to the scope in (c) targeting the MNM, the scope in (d) is along the CNM at the INT level.
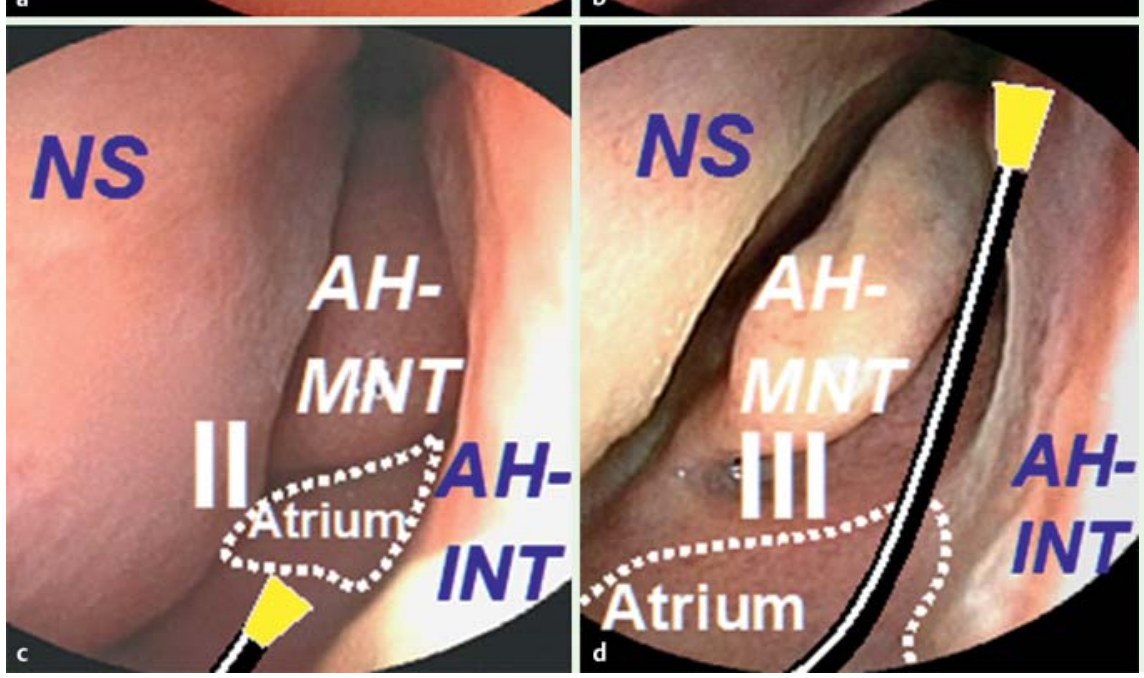

which was spacious enough to visualize both the posterior insertions of the MNT and INM (see the definition below) during anterior meatuscopy.

\section{Meatus selection principle for UT-EGD}

We inserted the scope through the "safest tunnel" (a grade III anterior-to-posterior CNM) of the nasal cavity as the first priority $(\bullet$ Fig. $2 \mathrm{~d}$ and $\bullet$ Fig.3d). We hypothesized that passing through this route would avoid injury to the INT or the MNT, which are the most common sites of epistaxis during transnasal endoscopy [20]. If this grade III anterior-to-posterior CNM route was not identified, the meatus with only a grade III INM or a grade III MNM was selected as a second choice.

Fewer patients had MNM evaluated with EMSS than INM or anterior CNM because the procedure is dependent upon grade III anterior CNMs. Thus all of the patients with MNM evaluable with EMSS were eligible for transnasal insertion and exertion. Adverse effects also were compared for patients undergoing endoscopy through a less than grade III MNM versus a less than grade III INM.

Three passes in anterior meatuscopy

Three endoscopic passes with anterior meatuscopy were performed on each side of the nasal cavity without the need for any local anesthetics or decongestant ( $\bullet$ Video 1 ).

First pass (for the INM) The scope was inserted a little behind the nasal vestibule, flexed downward, and gently passed along the

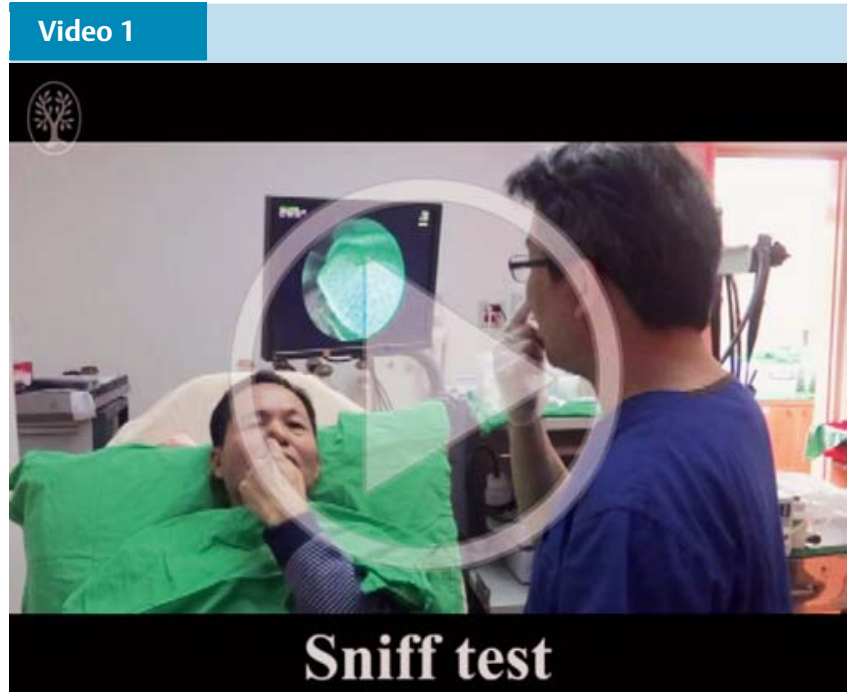

The author, Dr. C. Hu is demonstrating transnasal meatuscopy is more accurate than a sniff test to select a nostrile insertion route. Online content including video sequences viewable at: http://dx.doi.org/10.1055/s-00341392772 


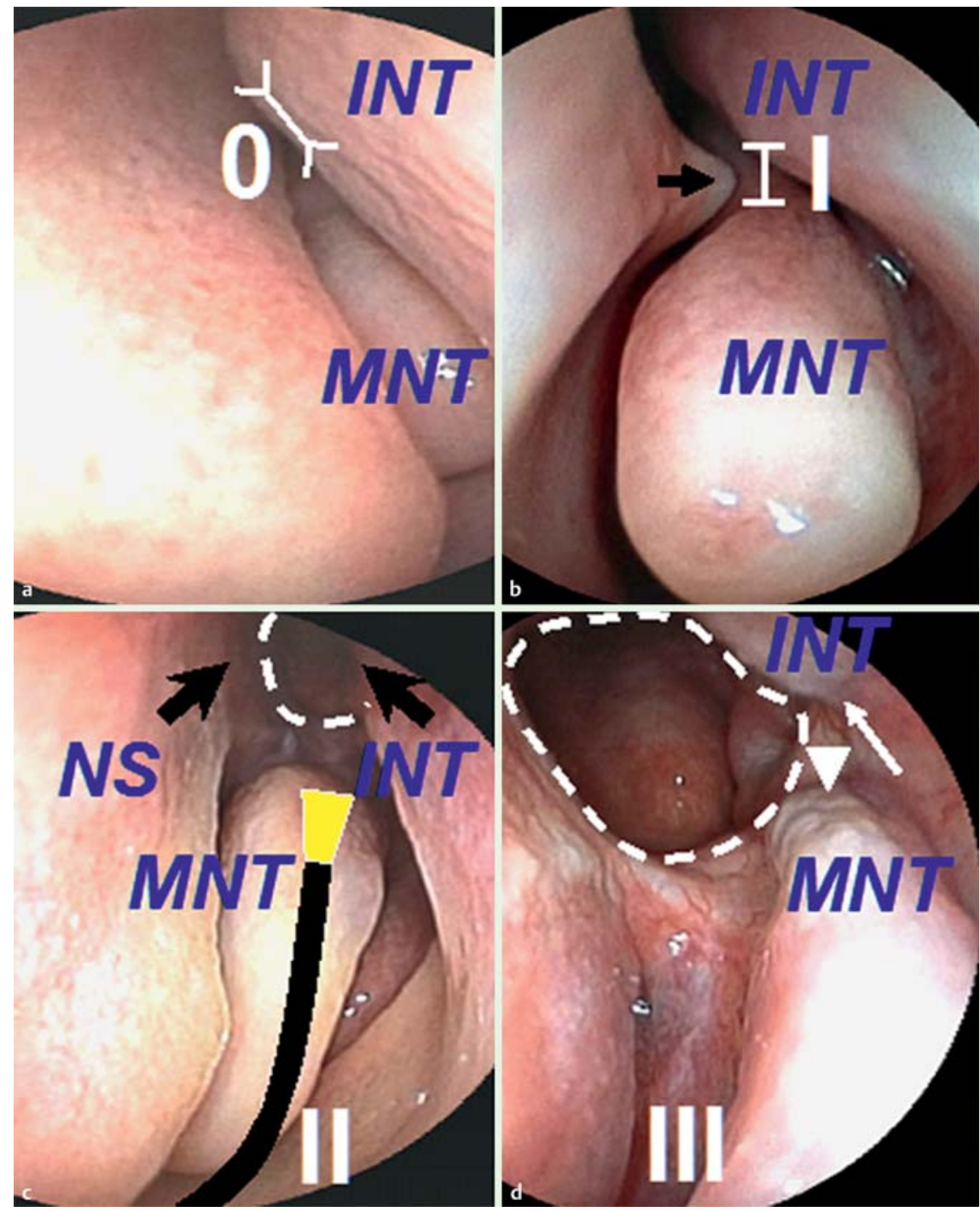

Fig. 4 EMSS of the MNM (right). The MNM is a canal situated between the MNT and the INT. (a) grade 0: no space; (b) grade I: slit-like distant MNM is seen; a septal spur (arrow) further narrows the MNM; (c) grade II: (i) the whole MNT and (ii) the incomplete choana (dotted line) are seen; further approach is prevented by scope resistance and/or nasal discomfort. An "endoscopic clamp" is formed by the INT and the nasal septum. The scope in (c) is along the inferior surface of the MNT; (d) grade III: (i) the posterior insertion of the MNT (PI-MNT, arrowhead), (ii) the PI-INT (arrow) and (iii) the complete choana (dotted circle) are seen.

INM until scope resistance and/or nasal discomfort was encountered. The far end of the posterior INM was usually inaccessible without decongestive anesthesia.

Second pass (for the anterior CNM) After observing the INM in the first pass, the endoscopist withdrew the scope to the anterior head of the INT, re-straightened the scope to view the entrance of the anterior CNM, and gently inserted the scope to the atrium of the MNM until scope resistance and/or nasal discomfort was encountered.

Third pass (for the MNM) The MNM was evaluable when the scope could be inserted to the atrium of the MNM without scope resistance and/or nasal discomfort. In the atrium of the MNM, the endoscopist reflexed the scope downward and gently passed it along the inferior border of the MNT towards the posterior MNM until scope resistance and/or nasal discomfort was encountered.

\section{Endoscopic meatus scoring scale}

During anterior meatuscopy, we used a simple endoscopic meatus scoring scale (EMSS) to define patencies of the INM, CNM, and MNM. A grade III meatus was defined as endoscopically patent. The definitions of each EMSS were defined as follows:
EMSS of the INM

The INM is the space between the inferior aspect of the INT and nasal floor (INT/NF: the "|" represents INM): grade 0: no space ( $\bullet$ Fig. 2a); grade I: only up-sloping INT border (black arrow) and slit-like distant INM were seen ( $\bullet$ Fig. $\mathbf{2 b}$ ); grade II: (i) the complete upsloping-downsloping INT border (curved scope route) with the transition angle $\left({ }^{*}\right)$ and (ii) the incomplete choana (dotted line) were seen; a nasal septum (NS) spur (arrow) abutting against the posterior MNM ( $\bullet$ Fig. 2 c); grade III: (i) the posterior insertion of the INT (PI-INT, arrow), (ii) the PI-MNT (arrowhead) and (iii) the complete choana (dotted circle) were seen $(\bullet$ Fig. 2d).

\section{EMSS of the anterior CNM}

The anterior CNM is the space between the NS and anterior head of the INT (AH-INT) (NS-INT: the "-" represents CNM): grade 0: no

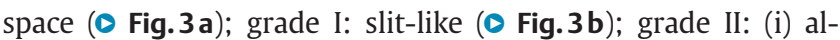
though the anterior head of the MNT (AH-MNT) was seen, the scope could not reach the atrium of the MNM (dotted line region) due to scope resistance and/or nasal discomfort $($ Fig. 3 c). Thus, with a grade II anterior CNM, EMSS of the inner MNM was regarded as not evaluable; grade III: the scope could reach the atrium of the MNM without scope resistance and/or nasal discom- 


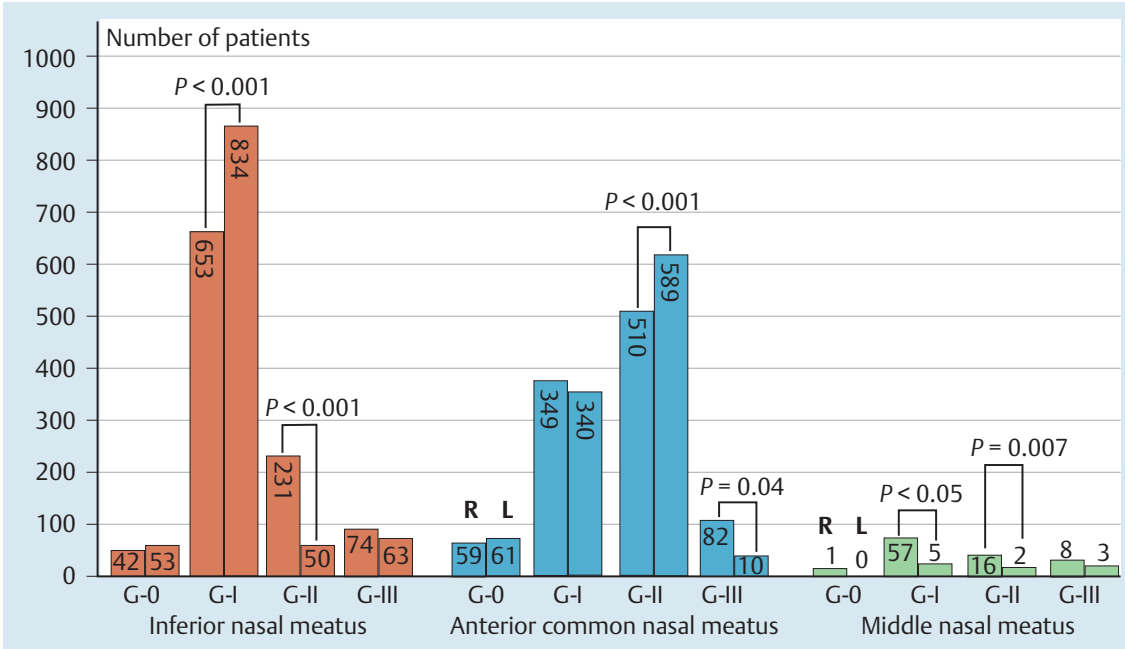

Fig. 5 EMSS frequencies of the nasal meatuses. $G=$ grade, $R=$ right, $L=$ left.

\begin{tabular}{|c|c|c|c|}
\hline & \multicolumn{3}{|c|}{ Anterior meatuscopy (gold standard) } \\
\hline & Right side more patent & Left side more patent & \\
\hline Right side more patent & 339 (TP) & 152 (FP) & PPV $69.1 \%$ \\
\hline \multicolumn{4}{|l|}{ Sniff test } \\
\hline \multirow[t]{2}{*}{ Left side more patent } & 196 (FN) & $313(\mathrm{TN})$ & NPV $61.4 \%$ \\
\hline & Sensitivity $63.30 \%$ & Specificity $67.30 \%$ & $\begin{array}{l}\text { Accuracy } 65.2 \\
\%\end{array}$ \\
\hline
\end{tabular}

Table 1 Sensitivity, specificity, PPV, and NPV of sniff test.

PPV, positive predictive value; NPV, negative predictive value; TP, true positive; FP, false positive; FN, false negative; TN, true negative

fort. Usually the whole AH-MNT was seen in a grade III anterior CNM ( Fig. 3 d).

\section{EMSS of the MNM}

The MNM is a canal situated between the MNT and the INT (MNT/ INT: the "/" represents MNM): grade 0: no space ( $\bullet$ Fig.4a); grade I: a slit-like posterior MNM was seen; a septal spur further narrowed the MNM ( $\bullet$ Fig.4b, arrow); grade II: (i) the whole MNT and (ii) the incomplete choana (dotted line) were seen; further approach was/were prevented by scope resistance or/and nasal discomfort ( $\bullet$ Fig.4c); grade III: (i) the posterior insertion of the MNT (PI-MNT, arrowhead), (ii) the PI-INT (arrow) and (iii) the complete choana (dotted circle) were seen $(\bullet$ Fig. $4 \mathrm{~d}$ ).

\section{Mnemonics for grading the three meatuses}

The mnemonic for using this four-grade scale for each meatus was easily remembered: (a) grade "0" indicated a meatus had N"O" visible space; (b) grade "I" indicated a meatus was SL"I"Tlike in width; (c) grade "II" indicated a meatus was about the width of a transnasal T"II"BE (i.e. endoscopic tube $\geqq$ meatus, scope not smoothly insertable), (d) grade "III" indicated the width of a meatus was more than that of a transnasal T"II"BE (i. e. endoscopic tube < meatus, scope smoothly insertable). Thus, a grade II meatus prevented the transnasal endoscope from entering the posterior meatuses due to scope resistance and/or nasal discomfort.

\section{Nasal anesthesia}

Gauze pledgetting was used to administer nasal anesthesia to all patients, in a $4: 1$ mixture of $4 \%$ lidocaine and 1:1000 epinephrine for decongestion as previously described [15]. During anterior meatuscopy, no local anesthetics were needed.

\section{Transnasal endoscopy}

Five minutes after decongestive anesthesia was administered, all patients underwent transnasal endoscopy administered by one experienced transnasal endoscopist (Doctor C. Hu) to avoid inter-endoscopist bias. All of the meatus insertion routes were selected according to the results of each patient's anterior meatuscopy. As mentioned above, one of the three routes including (i) INM ( $\bullet$ Fig. 2c), (ii) anterior-to-posterior CNM ( $\bullet$ Fig. $2 \mathrm{~d}$ ), and (iii) CNM-atrium-MNM ( $\bullet$ Fig. $3 \mathrm{c}$ and $\odot$ Fig. $4 \mathrm{c}$ ) was chosen according to the meatus selection principle to compare the adverse effects elicited during endoscopic passage through these three routes.

\section{Statistical analysis}

Descriptive statistics were used. The sensitivity, specificity, positive predictive value (PPV), negative predictive value (NPV) and accuracy of a sniff test to predict the more patent nostril were calculated using the results of anterior meatuscopy coupled with EMSS as the gold standard. A chi-square test was used to analyze the equivalence for ordinal variables. A $P$ value $<0.05$ was considered significant. Analyses were performed using SPSS 12.0.1C (SPSS Inc., Chicago, IL, USA). 


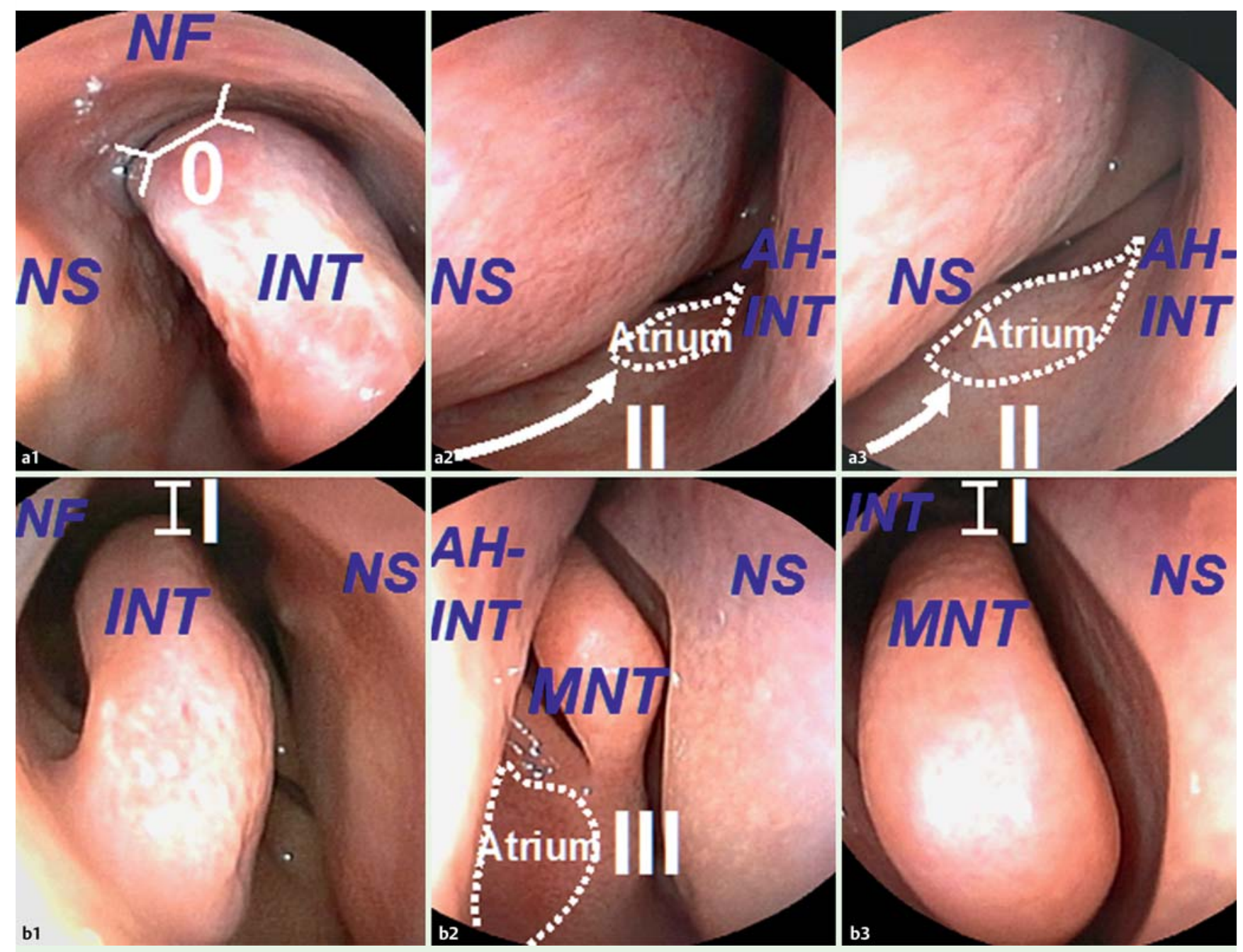

Fig. 6 omparison of the EMSS of both nasal cavities. On the right side: (A1) the INM is grade 0; (A2) the anterior CNM is grade II; (A3) the atrium (dotted-line regions from $A 2$ to $A 3$ ) is not approachable because of resistance to further scope insertion (white arrows from A2 to A3). Thus the EMSS of the inner MNM is regarded as not evaluable. On the left side: (B1) the INM is grade I; (B2) the atrium is approachable and thus the anterior CNM is grade III; (B3) the MNM is grade I. Compared with the right side (0, II, not evaluable), the left nasal meatuses (I, III, I) should be chosen for nasal anesthesia and endoscopic insertion.

\section{Results}

$\nabla$

\section{Patient characteristics}

Using anterior meatuscopy to select the most patent meatus for nasal anesthesia and insertion, we were able to complete UTEGD in all 1,000 cases (467 male and 533 female; mean age 54.9 years, range 19-92 years) without insertion failure.

\section{EMSS frequency distributions}

The EMSS frequencies of the three nasal meatuses in both sides (right $v s$. left) of the nasal cavity were compared. In the INM (grade I: 653 vs. 834, $P<0.001$; grade II: 231 vs. 50, $P<0.001$ ), anterior CNM (grade III: 82 vs. $10, P=0.04$ ), and MNM (grade I: 57 vs. $5, P<0.05$; grade II: 16 vs. $2, P=0.007$ ) significant differences were found between the EMSS frequencies on the right and left sides. Taking the right and left sides together, in the INM (grade $\mathrm{I}, \mathrm{n}=1487>$ grade $\mathrm{II}, \mathrm{n}=281>$ grade $\mathrm{III}, \mathrm{n}=137>$ grade $0, \mathrm{n}$ =95) and MNM (grade I, $\mathrm{n}=62>$ grade II, $\mathrm{n}=18>$ grade III, $\mathrm{n}=11>$ grade $0, n=1$ ), the EMSS frequencies were both on the order of grade $\mathrm{I}>\mathrm{II}>\mathrm{III}>0$. For the anterior CNM (grade II, $\mathrm{n}=1099>$ grade I, $n=689>$ grade $0, n=120>$ grade III, $n=92$ ), the EMSS frequencies were on the order of grade II $>$ I $>0>$ III $(\varangle$ Fig. 5 ).
Sniff test was not reliable

With a sniff test, a patient might mistakenly select the right nose, whereas the EMSS could confirm that the left meatuses were more patent than the right ones ( Video 1 and $\bullet$ Fig. 6 ), and vice versa. Using EMSS as the gold standard, we found that a sniff test was not reliable, given its low sensitivity (63.3\%), specificity (67.3\%), PPV (69.1\%), NPV (61.4\%), and accuracy (65.2\%). With anterior meatuscopy, 320.7 out of 1,000 cases (32.1\%) were redirected to use the left nostril when a sniff test falsely directed the endoscopist to use the right nostril. Similarly, 360.6 out of 1,000 cases $(36.1 \%)$ were redirected to use the right nostril when a sniff test wrongly directed the endoscopist to use the left nostril (๑ Table 1$)$.

The CNM was endoscopically patent only in about $10 \%$ of cases

To fulfill the criterion of an endoscopically patent CNM at the INT level, EMSS for both the anterior CNM and the INM should be grade III. Accordingly, 137 cases had grade III INM and 92 cases had grade III anterior CNM ( $\bullet$ Fig. 5 ). In all 92 cases with a grade III anterior CNM, the INM was simultaneously grade III. Thus, 9.2 $\%(92 / 1,000)$ of our patients had spacious anterior-to-posterior 


\begin{tabular}{|c|c|c|c|}
\hline & Grade III ( $n=92)$ & Grade III $(n=45)$ & $P$ value $(95 \% \mathrm{Cl})$ \\
\hline & CNM, n (\%) & INM only, n (\%) & \\
\hline Epistaxis & 0 & $3(6.7)$ & $0.058(-0.6-14.0)$ \\
\hline Nasal pain & 0 & $5(11.1)$ & $0.006(1.9-20.3)$ \\
\hline Nasal discharge & $3(3.3)$ & $8(17.8)$ & $0.009(2.7-26.3)$ \\
\hline \multirow[t]{3}{*}{ Total } & $3.3 \%$ & $35.6 \%$ & $<0.0001(17.8-46.8)$ \\
\hline & Grade III $(\mathrm{N}=92)$ & Grade III $(\mathrm{N}=11)$ & $P$ value $(95 \% \mathrm{Cl})$ \\
\hline & CNM, n (\%) & MNM only, $n$ (\%) & \\
\hline Epistaxis & 0 & $1(9.1)$ & $0.02(0.7-17.5)$ \\
\hline Nasal Pain & 0 & $3(27.3)$ & $<0.001(0.9-53.6)$ \\
\hline Nasal discharge & $3(3.3)$ & $4(36.4)$ & $<0.001(22.3-50.5)$ \\
\hline \multirow[t]{3}{*}{ Total } & $3.3 \%$ & $72.8 \%$ & $<0.0001(56.0-83.0)$ \\
\hline & Grade III (N = 45) & Grade III (N=11) & $P$ value $(95 \% \mathrm{Cl})$ \\
\hline & INM only, $n(\%)$ & MNM only, n (\%) & \\
\hline Epistaxis & $3(6.7)$ & $1(9.1)$ & $0.71(-16.1-20.9)$ \\
\hline Nasal Pain & $5(11.1)$ & $3(27.3)$ & $0.37(-11.7-44.1)$ \\
\hline Nasal discharge & $8(17.8)$ & $4(36.4)$ & $0.45(-11.9-49.2)$ \\
\hline \multirow[t]{3}{*}{ Total } & $35.6 \%$ & $72.8 \%$ & $0.05(7.4-66.9)$ \\
\hline & Grade $0 \sim \mathrm{II}(\mathrm{N}=771)$ & Grade $0 \sim$ II $(\mathrm{N}=81)$ & $P$ value $(95 \% \mathrm{Cl})$ \\
\hline & INM, n (\%) & MNM, n (\%) & \\
\hline Epistaxis & $74(9.6)$ & $9(11.1)$ & $0.81(-5.7-8.7)$ \\
\hline Nasal Pain & $278(36.1)$ & $34(41.9)$ & $0.36(-5.5-17.1)$ \\
\hline Nasal discharge & 308 (39.9) & $36(44.4)$ & $0.51(-6.9-15.9)$ \\
\hline Total & $85.6 \%$ & $97.4 \%$ & $0.005(7.5-16.1)$ \\
\hline
\end{tabular}

CNM, common nasal meatus; INM, inferior nasal meatus; MNM, middle nasal meatus

CNMs being used for endoscopic insertion and exertion. In contrast, $90.8 \%(908 / 1,000)$ of our patients received nasal insertion via the INM or the MNM.

Passing through the CNM, if endoscopically patent, reduced complications

Adverse effects were compared for the 92 patients (with a grade III anterior-to-posterior CNM), the 45 (137-92) with only a grade III INM, and the 11 patients with only a grade III MNM. Passing through the CNM versus the INM and the CNM versus the MNM produced marginal differences in reduction of epistaxis $(P$ $=0.058$ and $P=0.02$, respectively), but the rates of nasal pain $(P=$ 0.006 and $P<0.001)$ and nasal discharge $(P=0.009$ and $P<0.001$, respectively) were significantly decreased. Most importantly, adoption of the anterior-to-posterior grade III CNM was associated with complete freedom from epistaxis $(\mathrm{n}=0)$ and nasal pain $(n=0)$. However, endoscopic passage through the INM rather than the MNM with equivalent grade III patency scores was associated with a trend toward reduction in total adverse effects but the difference did not rise to statistical significance $(P=0.05)$. However, compared with patients with grade 0-II INMs, those with grade 0-II MNMs for transnasal passage had significantly more adverse effects overall $(P=0.005)$ ( $\bullet$ Table 2$)$.

\section{Discussion}

\section{Dis}

This study confirmed that anterior meatuscopy is more reliable than a sniff test for predicting nasal patency before UT-EGD. By comparing grading scales between the right and left meatuses, a transnasal endoscopist can be confident in applying nasal anesthesia and inserting to the meatus of choice while minimizing complications. We proposed reporting the how, where, and grading of nasomucosal injury after transnasal endoscopy and em- phasized that a grading scale for defining insertion difficulty through a nasal meatus is important because it may be correlated with the severity of nasomucosal injury and bleeding [16]. Since making those recommendations, we have been advocating anterior meatuscopy to compare meatus patency on both sides of the nasal cavity. Although the nasal cavity is a complex compartment, we used simple anatomical landmarks, patient feedback, and endoscope resistance to define EMSS (grade 0, I, II, and III). To our knowledge, this is the first endoscopic method studied for assessing meatus patency before UT-EGD.

Given that a sniff test is not only unreliable but also cannot be performed in patients who are unable to sniff, anterior meatuscopy is an attractive alternative. Using anterior meatuscopy, we revealed that the anterior INM is broader than the posterior INM, whereas the anterior MNM is narrower than the posterior MNM; these endoscopic observations are compatible with a cadaveric anatomical study [19]. We have also found that the anterior CNM is narrower than the posterior CNM, which is also consistent with an anatomical study demonstrating that the INT bone is thicker anteriorly than posteriorly [20].

With anterior meatuscopy, we have revealed that the INT has a more prominently convoluted shape than the MNT, comprising an up-sloping INT border, a transition angle, and a down-sloping INT border. In contrast, the CNM-atrium-MNM tract is more straightforward. Therefore, nasal anesthesia, either by the cotton-tipped applicator method [14], gauze pledgetting [15] or the Japanese catheter method [21], most often targets the unswerving MNM route. The anatomy may also explain why the majority of cases of epistaxis $(78 \%)$ reported by Mori et al occurred in the MNM [18].

Endoscopically the superior nasal turbinate and its meatus are hardly seen, and thus, have been called the forgotten turbinate [22]. Similarly, most endoscopists recognize the INM and MNM but are ignorant of the CNM. With anterior meatuscopy, we have 
found that the CNM is wider at the INT level than that at the MNT level. Most importantly, we found that endoscope insertion and exertion through an endoscopically patent CNM (grade III), if pre-confirmed by anterior meatuscopy, may avoid epistaxis and nasal pain, and minimize nasal discharge.

We perform anterior meatuscopy before transnasal endoscopy but it is also suitable for transnasal laryngoscopy, esophagoscopy, endoscopic surgery, and bronchoscopy. Anterior meatuscopy should be performed prior to these transnasal endoscopic procedures because nasonasal reflexes and the physiological nasal cycle may influence meatus patency on either side of the nasal cavity from time to time [23]. In our study, the EMSS frequencies of the INM and MNM (grade I $>$ II $>$ III $>0$ ) and that of the anterior $\mathrm{CNM}$ (grade $\mathrm{II}>\mathrm{I}>0>\mathrm{III}$ ) were found to be heterogeneous and variable. However, with EMSS, we have found that most of our patients have narrow meatuses (grades I II). Thus in Taiwanese and probably in all Asian patients, the predominance of lowgrade EMSS justifies anterior meatuscopy before UT-EGD for selection of the most patent meatus and administration of meticulous nasal anesthesia.

Meatuscopy is a simple procedure that can be used on patients of different races, especially those with narrow nasal tracts. In the future, it may serve as a more objective measure of meatus patency before any transnasal endoscopic procedure than a sniff test. As anterior meatuscopy gains in popularity, the role of the sniff test may become diminished. The purpose of "sniff" will not be as a "test" for selecting the nostril but for inhaling decongestants and anesthetics before UT-EGD.

Although ours was a retrospective study, the large number of cases may make the data representative for Asian patients. The meatuscopic findings, such as the most safety route in the CNM, endoscope clamp in the MNM, and the parabolic route in the INM, are noteworthy observations found by successive anterior meatuscopy. These novel concepts may make transnasal endoscopy more understandable and attractive to many endoscopists. This is the first epidemiologic study to reveal the patency scores of the INM, CNM, and MNM. However, more studies are needed to further elucidate the usefulness of anterior meatuscopy before UT-EGD in Western and European countries. In conclusion, a sniff test is not reliable whereas anterior meatuscopy can be used to objectively select the most patent meatus insertion site to avoid complications. Endoscope passage through the endoscopically patent CNM, if pre-evaluated by anterior meatuscopy, may avoid epistaxis and nasal pain, and minimize nasal discharge.

\section{Competing interests: None}

\section{References}

1 Choe WH, Kim JH, Ko SY et al. Comparison of transnasal small-caliber vs. peroral conventional esophagogastroduodenoscopy for evaluating varices in unsedated cirrhotic patients. Endoscopy 2011; 43: 649-656

2 Sami SS, Dunagan KT, Johnson ML et al. A Randomized Comparative Effectiveness Trial of Novel Endoscopic Techniques and Approaches for Barrett's Esophagus Screening in the Community. Am J Gastroenterol 2015; 110: $148-158$
3 Nevah MI, Lamberth JR, Dekovich AA. Transnasal PEG tube placement in patients with head and neck cancer. Gastrointest Endosc 2014; 79: 599-604

4 Wildi SM, Gubler C, Vavricka SR et al. Transnasal endoscopy for the placement of nasoenteral feeding tubes: does the working length of the endoscope matter? Gastrointest Endosc 2007; 66: 225 -229

5 Itoi T, Kawai T, Sofuni A et al. Efficacy and safety of 1-step transnasal endoscopic nasobiliary drainage for the treatment of acute cholangitis in patients with previous endoscopic sphincterotomy (with videos). Gastrointest Endosc 2008; 68: 84-90

$6 \mathrm{Hu} \mathrm{CT}$. Transnasal pharyngoscopy to assist nasobiliary tube placement: a simple technique to avoid injury to the patient, endoscopist, and endoscope. Endoscopy 2011; 43: 923-924

7 Lin J, Chen M, Lei $W$ et al. Eradication of diffuse gastric Peutz-Jeghers polyps by unsedated transnasal snare polypectomy and argon plasma coagulation. Endoscopy 2009; 41: E207-208

$8 \mathrm{Ai} Z \mathrm{ZL}$, Lan CH, Fan LL et al. Unsedated transnasal upper gastrointestinal endoscopy has favorable diagnostic effectiveness, cardiopulmonary safety, and patient satisfaction compared with conventional or sedated endoscopy. Surg Endosc 2012; 26: 3565-3572

9 Cohen JT, Safadi A, Fliss DM et al. Reliability of a transnasal flexible fiberoptic in-office laryngeal biopsy. JAMA Otolaryngol Head Neck Surg 2013; 139: 341 - 345

10 Sabirin J, Abd Rahman M, Rajan P. Changing trends in oesophageal endoscopy: a systematic review of transnasal oesophagoscopy. ISRN Otolaryngol 2013; 2013: 586973

11 Peery AF, Hoppo T, Garman KS et al. Barrett's esophagus risk consortium. Feasibility, safety, acceptability, and yield of office-based, screening transnasal esophagoscopy (with video). Gastrointest Endosc 2012; 75: $945-953$

12 Hoppo T, Jobe BA. Techniques of office-based endoscopy: unsedated transnasal endoscopy. Principles of flexible endoscopy for surgeons. 2013: $201-213$

13 Tatsumi Y, Harada A, Matsumoto T et al. Current status and evaluation of transnasal esophagogastroduodenoscopy. Dig Endosc 2009; 21: $141-146$

$14 \mathrm{Hu}$ CT. Endoscopic-guided versus cotton-tipped applicator methods of nasal anesthesia for transnasal esophagogastroduodenoscopy: a randomized, prospective, controlled study. Am J Gastroenterol 2008; 103: $1114-1121$

$15 \mathrm{Hu} \mathrm{CT}$. Gauze pledgetting versus endoscopic-guided aerosolized spray for nasal anesthesia before transnasal esophagogastroduodenoscopy: a prospective, randomized study. Gastrointest Endosc 2010; 71: 11 20

$16 \mathrm{Hu} \mathrm{C}$. Reporting the how, where, and grading of nasomucosal injury after transnasal endoscopy. Endoscopy 2010; 42: 688-689

$17 \mathrm{Hu} \mathrm{CT}$. Nasal anesthesia and body position changes: lessons learned from self-performed, transnasal EGD. Gastrointest Endosc 2010; 71: $1277-1279$

18 Mori A. Reporting the how, where, and grading of nasomucosal injury after transnasal endoscopy (Letter reply to Hu). Endoscopy 2010; 42: 689

19 Dahlstrom K, Olinger A. Anatomic description of the middle meatus and classification of the hiatus semilunaris into five types based upon morphological characteristics. Clin Anat 2014; 27: 176-181

20 Proetz AW. Physiology of the nose from the standpoint of the plastic surgeon. Arch Otolaryngol 1944; 39: 514-517

21 Lee YS, Bang CS, Suk KT et al. Catheter-free method is sufficient for preparation for transnasal endoscopy: randomized controlled trial. Dig Endosc 2014; 26: 385-391

22 Clerico DM. Pneumatized superior turbinate as a cause of referred migraine headache. Laryngoscope 1996; 106: 874-879

23 Baraniuk JN, Kim D. Nasonasal reflexes, the nasal cycle, and sneeze. Curr Allergy Asthma Rep 2007; 7: 105-111 